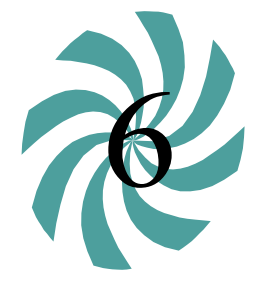

Tecnociencia, Vol. 22, N²: 103-121

julio-diciembre 2020

\title{
SARCOPHAGIDAE DE INTERÉS FORENSE EN EL PARQUE NACIONAL SOBERANÍA, PROVINCIA DE PANAMÁ
}

\author{
${ }^{1}$ Percis A. Garcés, ${ }^{2}$ Litza N. Arias \& Meybis Medina \\ ${ }^{1}$ Universidad de Panamá, Vicerrectoría de Investigación y Postgrado, Programa \\ Centroamericano de Maestría en Entomología, \\ ${ }^{2}$ Ministerio de Educación \\ e-mail 1'perchysg@gmail.com; ${ }^{2}$ nadily10@gmail.com
}

\section{RESUMEN}

Se estudiaron las Sarcophagidae en dos áreas: una boscosa y en otra no boscosa, con el propósito de conocer las especies que pudieran tener importancia forense en nuestro país. Esta familia contiene algunas especies que han sido registradas como insectos forenses importantes, debido a que son uno de los primero que detecta y encuentra un cadáver fresco. Por lo que, resultan importantes en las investigaciones criminales, principalmente en la estimación del intervalo postmortem (IPM). En el presente estudio se colectaron 169 ejemplares que fueron agrupados en nueve géneros y 11 especies. Las especies más frecuentemente capturadas fueron, Pekia (Pantonella) intermutans, Sarcodexia sp, Boettcheria sp, Pekia sp, Helicobia sp y Sarcofahrtiopsis $\mathrm{sp} 2$. En cuanto a la preferencia de las áreas, en nuestro estudio, las moscas mostraron mayor preferencia por el área boscosa que por el área no boscosa y, por el corazón que por el hígado.

\section{PALABRAS CLAVES}

Sarcophagidae, área boscosa, área no boscosa, Pekia (Pantonella) intermutans, Sarcodexia sp, Boettcheria sp. 


\title{
SARCOPHAGIDAE OF FORENSIC INTEREST IN THE SOBERANIA NATIONAL PARQUE, PROVINCE OF PANAMA
}

\begin{abstract}
Sarcophagidae were studied in two areas: wooded and non-wooded with the purpose to learn about species that could have forensic importance in our country. This family has some species that have been registered as important forensic insecto, because they are some of the are important in criminal investigation, specially to estimate postmortem interval (IPM). In this study 169 specimenes were collected and grouped in 9 genera and 11 species. The most frequent species captured were: Pekia (Pantonella) intermutans, Sarcodexia sp, Boettcheria sp, Pekia sp, Helicobia sp y Sarcofahrtiopsis sp2. Referring to the áreas preference for the woody área than for the non-woody area, and for the heart tissue tan for el liver.
\end{abstract}

\section{KEY WORDS}

Sarcophagidae, wooded area, non wooded area, Pekia (Pantonella) intermutans, Sarcodexia sp, Boettcheria sp.

\section{INTRODUCCIÓN}

La familia Sarcophagidae es la segunda más grande dentro de la superfamilia Oestroidea (McAlpine, 1981), con aproximadamente 171 géneros y 3094 especies (Pape et al. 2011), incluidas en las subfamilias Miltogramminae, Paramacronychiinae y Sarcophaginae, siendo esta última la más diversa en la Región Neotropical con alrededor de 780 especies (Pape, 1996).

Estas moscas pueden ser reconocidas por la presencia de un patrón general de coloración gris muy uniforme, con tres franjas longitudinales negras en el mesonotum y abdomen a cuadros o manchados; la presencia de una fila de setae en el meron y el subscutelum subdesarrollado. La mayoría de las especies tienen un tamaño medio a grande $(8-25 \mathrm{~mm})$, pero hay pocas especies más pequeñas $(5-8 \mathrm{~mm})$ (Shewell, 1987).

Las Sarcophagidae son elementos muy importantes del componente 
necrófago, debido a que ciertas especies aparecen frecuentemente en cadáveres humanos (Goff, 1991; Anderson, 1995; Oliva, 1997), siendo un grupo pionero en la sucesión entomológica en cadáveres humanos, por lo que se les considera indicadores relevantes del Intervalo postmortem (IPM) (Wells et al., 2001; Camacho, 2005; Pérez et al., 2005; Buenaventura et al., 2009; Buenaventura y Pape, 2013).

Las Sarcophagidae poseen la característica de depositar larvas de primer estadio sobre los sustratos de cría (Barros et al., 2008; Pape y Dahlem, 2010). Esta característica les confiere cierta ventaja comparativa en comparación con las especies ovíparas, en especial cuando se trata de sustratos efímeros, como son los casos de pequeños cadáveres o cadáveres en condiciones de rápida desecación (Barros et al., 2008; Pape y Dahlem, 2010).

Este estudio tuvo como objetivos: 1) Determinar las especies de Sarcophagidae que primeramente son atraídas a los tejidos de hígado y corazón de cerdos domésticos, 2) comparar las especies de moscas que habitan en un área boscosa y en una no boscosa del Parque Nacional Soberanía.

\section{MATERIALES Y MÉTODOS}

El Parque Nacional Soberanía (PNS) creado en 1980, se encuentra ubicado entre las provincias de Panamá y Colón, al sureste de la Cuenca Hidrográfica del Canal de Panamá, se encuentra a solo 20 minutos de la capital y 40 minutos de la ciudad de Colón, sus coordenadas geográficas se encuentran entre los $9^{\circ} 04^{\prime} 27^{\prime \prime} \mathrm{N}$ y $79^{\circ} 39^{\prime} 35^{\prime \prime} \mathrm{O}$. Los cerros que dominan su topografía cuya altitud máxima es de $85 \mathrm{msnm}$ e incluye 22,104 hectáreas de bosque tropical, con temperatura promedio de $25^{\circ} \mathrm{C}$ y una humedad relativa de aproximadamente $80 \%$, con precipitaciones anuales promedio de $2131 \mathrm{~mm}$ por año (Mida, 1985).

Se seleccionaron dos sitios representativos del PNS que comprenden el área boscosa y una no boscosa. El área boscosa se caracterizó por presentar una vegetación con diversos tipos de árboles frondosos como el ceibo, (Ceiba pentandra), el Espavé (Anacardium excelsum), el Cuipo 
(Cavanillesia platanifolia) el Árbol Panamá (Sterculea apetala) que alcanzan unos 20 a 30 metros de altura. Mientras que el área no boscosa predomina una vegetación arbustiva entremezclada con pastizales, donde se observan parches de bosque en proceso de recuperación como resultado de la intervención humana, especialmente la carretera Gaillard llamada Vía Forestal, ambos sitios de muestreo estaban aproximadamente $50 \mathrm{~m}$ de distancia uno del otro.

Para la captura de los ejemplares adultos se emplearon dos tejidos de cerdo (Sus scrofa) (hígado y corazón) a $12 \mathrm{~h}$ horas después de su extracción. Se cortaron en fragmentos de $200 \mathrm{~g}$ el hígado y $110 \mathrm{~g}$ el corazón, atendiendo a los tamaños de ambos órganos. Los mismos se colocaron en sus respectivos recipientes plásticos, herméticamente cerrados y debidamente rotulados. Posteriormente fueron expuestos en las áreas boscosa y no boscosa, donde también se registraron las temperaturas de los tejidos y la temperatura ambiental, siguiendo la metodología de Pineda (2011). Se realizaron dos pseudoréplicas con ambos tejidos y se partieron en dos partes iguales para ser colocadas en cada área de estudio y distribuidos a una distancia de $5 \mathrm{~m}$ entre los tejidos $\mathrm{y}$ de $30 \mathrm{~m}$ entre los sitios.

Los tejidos fueron expuestos en intervalos de tiempo de 12, 24, 48, 72 y 96 horas de descomposición. Cada muestreo fue realizado durante tres horas continuas, de 9:00 am a 12:00 m.d. Los intervalos de las tres horas, fueron primeramente divididos en intervalos de 10 minutos, hasta completar la primera hora de muestreo. La segunda hora de muestreo, se dividió en intervalos de 15 minutos. Finalmente, la tercera hora de muestreo, se dividió intervalos de 20 minutos, hasta completar la tercera hora. Las capturas de las moscas se realizaron con la ayuda de una red entomológica. Las muestras recolectadas fueron trasladas a los laboratorios de la Universidad de Panamá para su identificación.

Los ejemplares de Sarcophagidae fueron separaron e identificados con la clave taxonómica de (Buenaventura et al., 2009).

Este estudio es de tipo descriptivo y tuvo como propósito establecer el orden en que llegaron las principales especies de moscas Sarcophagidae 
que fueron atraídas a los tejidos de cerdos domésticos (hígado y corazón).

\section{RESULTADOS}

Se colectaron 169 ejemplares que estuvieron distribuidos en nueve géneros y 11 especies. Las especies más frecuentemente capturadas, fueron Pekia (pantonella) intermutans, el 27.90\%, (46 especímenes), Sarcodexia $s p$, con el $26.70 \%$, (43 especímenes), Boettcheria $s p$ con el 12.2\%, (20 especímenes, Peckia sp con el 10.4\%, (17 especímenes) y Helicobia $s p$ con el 9.2\% (15 especímenes) (Figura 1).

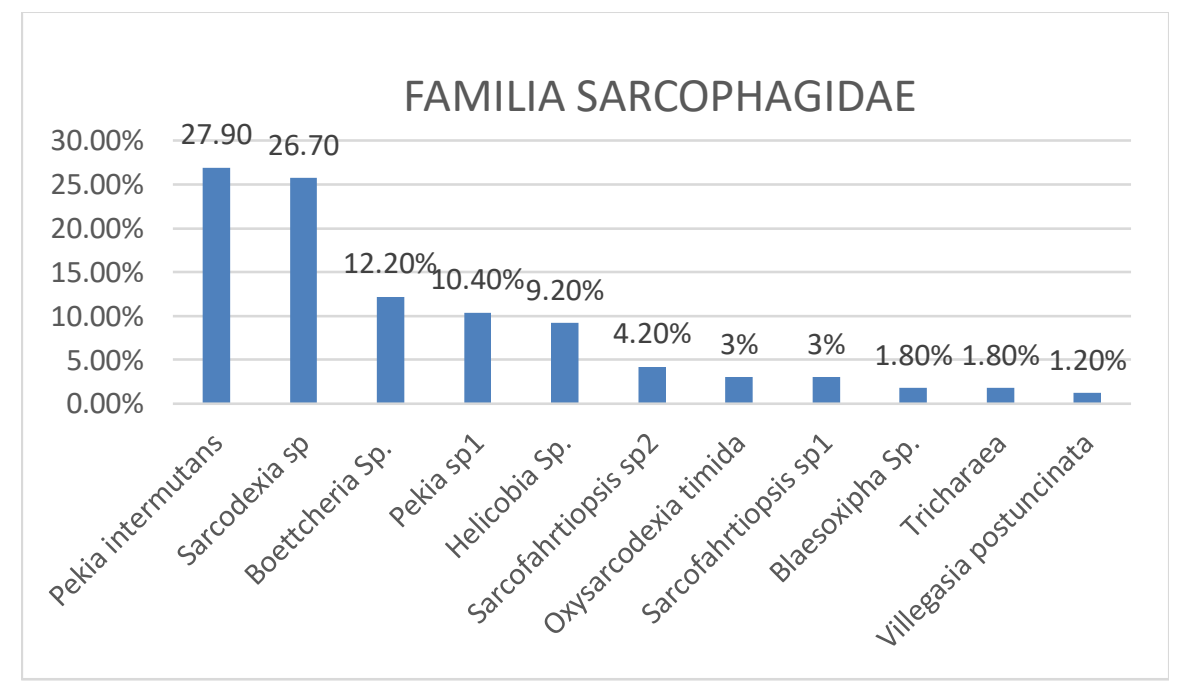

Fig. 1 Especies de Sarcophagidae más frecuentemente capturadas

En cuanto a las áreas muestreadas, en el área boscosa se capturaron 92 especímenes, en tanto que en el área no boscosa 78. Las especies que predominaron en el área boscosa fueron, Pekia (pantonella) intermutans con el 22.8\%, seguido de Sarcodexia sp. con el 25.0\%, Helicobia sp con el 10.8\%, Boettcheria sp. y Peckia sp. con el 9.7\% respectivamente. En tanto que en el área no boscosa predominaron las mismas especies Pekia (pantonella) intermutans con el 35.8\%, seguido de Sarcodexia sp. con el $27.2 \%$, Boettcheria sp. con el 16.2\%, y Peckia 
sp. con el 8.9\% (Cuadro 1).

Cuadro 1. Comparación de especies capturadas en área boscosa y no boscosa del Parque Nacional Soberanía (PNS)

\begin{tabular}{|l|r|r|r|r|r|}
\hline \multicolumn{1}{|c|}{ Especies } & $\begin{array}{c}\text { Área } \\
\text { Boscosa }\end{array}$ & $\mathbf{( \% )}$ & $\begin{array}{c}\text { Área no } \\
\text { boscosa }\end{array}$ & (\%) & Total \\
\hline SARCOPHAGIDAE & & & & & \\
\hline $\begin{array}{l}\text { Pekia (pantonella) } \\
\text { intermutans }\end{array}$ & 21 & 22.8 & 28 & 35.8 & $\mathbf{4 9}$ \\
\hline Sarcodexia sp. & 23 & 25.0 & 21 & 27.2 & $\mathbf{4 3}$ \\
\hline Boettcheria sp. & 9 & 9.7 & 12 & 16.2 & $\mathbf{2 1}$ \\
\hline Pekia sp. & 9 & 9.7 & 7 & 8.9 & $\mathbf{1 6}$ \\
\hline Helicobia sp. & 10 & 10.8 & 5 & 6.4 & $\mathbf{1 5}$ \\
\hline Sarcofahtiopsis sp2 & 4 & 4.3 & 3 & 3.8 & $\mathbf{7}$ \\
\hline Sarcofahtiopsis sp1 & 5 & 5.4 & 0 & 0 & $\mathbf{5}$ \\
\hline Oxysarcodexia timida & 5 & 5.4 & 0 & 0 & $\mathbf{5}$ \\
\hline Blaexosipha sp. & 3 & 3.2 & 0 & 0 & $\mathbf{3}$ \\
\hline Tricharaea & 1 & 1.0 & 2 & 2.5 & $\mathbf{3}$ \\
\hline Villegasia puncinata & 2 & 2.1 & 0 & 0 & $\mathbf{2}$ \\
\hline & $\mathbf{9 2}$ & $\mathbf{1 0 0}$ & $\mathbf{7 8}$ & $\mathbf{1 0 0}$ & $\mathbf{1 6 9}$ \\
\hline
\end{tabular}

En cuanto a los tejidos, en el corazón se capturó 11 especies, mientras que en el hígado se capturaron nueve especies. En el corazón se capturaron 110 ejemplares, en tanto que en el hígado se capturaron 56 ejemplares. Las especies más abundantes en el corazón fueron Pekia (pantonella) intermutans con $27.2 \%$ especímenes, Sarcodexia sp. con $26.3 \%$ Boettcheria sp. con $12.7 \%$ y Helicobia sp. con $11.2 \%$ En tanto que en el hígado, las especies más frecuentes fueron las mismas mencionadas anteriormente, Pekia (pantonella) intermutans con 30.5\% especímenes y Sarcodexia sp. con $25.4 \%$, Boettcheria sp con $11.8 \%$, Peckia sp 14.2\% (Cuadro 2). 
Cuadro 2. Comparación de las especies capturadas en los tejidos (hígado y corazón)

\begin{tabular}{|l|r|r|r|r|r|}
\hline \multicolumn{1}{|c|}{ Especies } & Corazón & $\begin{array}{l}\text { Abundancia } \\
\text { relativa \% }\end{array}$ & Hígado & $\begin{array}{l}\text { Abundancia } \\
\text { relativa \% }\end{array}$ & Total \\
\hline SARCOPHAGIDAE & & & & & \\
\hline $\begin{array}{l}\text { Pekia (pantonella) } \\
\text { intermutans }\end{array}$ & 30 & 27.2 & 18 & 30.5 & $\mathbf{4 8}$ \\
\hline Sarcodexia sp. & 29 & 26.3 & 15 & 25.4 & $\mathbf{4 3}$ \\
\hline Boettcheria sp. & 14 & 12.7 & 7 & 11.8 & $\mathbf{2 1}$ \\
\hline Pekia sp. & 9 & 8.4 & 8 & 14.2 & $\mathbf{1 7}$ \\
\hline Helicobia sp. & 12 & 11.2 & 3 & 5.0 & $\mathbf{1 5}$ \\
\hline Sarcofahtiopsis sp2 & 4 & 3.6 & 3 & 5.0 & $\mathbf{7}$ \\
\hline Sarcofahtiopsis sp1 & 3 & 2.7 & 1 & 1.6 & $\mathbf{4}$ \\
\hline Oxysarcodexia timida & 3 & 2.7 & 3 & 5.0 & $\mathbf{6}$ \\
\hline Blaexosipha sp. & 3 & 2.7 & 0 & 0 & $\mathbf{3}$ \\
\hline Tricharaea & 1 & 0.9 & 2 & 3.3 & $\mathbf{3}$ \\
\hline Villegasia puncinata & 2 & 1.8 & 0 & 0 & $\mathbf{2}$ \\
\hline Total & $\mathbf{1 1 0}$ & $\mathbf{1 0 0}$ & $\mathbf{5 9}$ & $\mathbf{1 0 0}$ & $\mathbf{1 6 9}$ \\
\hline
\end{tabular}

A las 12 horas de exposición de los tejidos, en la primera hora de muestreo, no se capturó ninguna especie en los tejidos.

A las 24 horas de exposición de los tejidos, las principales especies que arribaron a los tejidos a la primera hora fueron Pekia (pantonella) intermutans con cinco especímenes y Pekia sp con dos (Cuadro 3).

Cuadro 3. Total de especies de moscas capturadas a las 24 horas en el pns

\begin{tabular}{|c|c|c|c|c|c|c|c|c|c|c|c|c|c|c|c|c|}
\hline \multirow[t]{2}{*}{ ESPECIES DE MOSCAS } & \multicolumn{6}{|c|}{$\begin{array}{l}\text { PRIMERA HORA } \\
\text { (MIN) }\end{array}$} & \multirow[t]{2}{*}{ TOTAL } & \multicolumn{4}{|c|}{$\begin{array}{c}\text { SEGUNDA } \\
\text { HORA } \\
\text { (MIN) }\end{array}$} & \multicolumn{4}{|c|}{$\begin{array}{cc}\text { TERCERA } \\
\text { TOTAL } \\
\begin{array}{c}\text { HORA } \\
(\mathrm{MIN})\end{array} \\
\end{array}$} & \multirow[t]{2}{*}{ TOTAL } \\
\hline & 10 & 20 & 30 & 40 & 50 & 60 & & 15 & 30 & 45 & 60 & & 20 & 40 & 60 & \\
\hline \multicolumn{17}{|l|}{ SARCOPHAGIDAE } \\
\hline $\begin{array}{l}\text { Pekia (pantonella) } \\
\text { intermutans }\end{array}$ & 2 & 0 & 0 & 1 & 1 & 1 & 5 & 0 & 0 & 0 & 0 & $\mathbf{0}$ & 1 & 0 & 0 & 1 \\
\hline Pekia sp1 & 1 & 0 & 0 & 1 & 0 & 0 & 2 & 0 & 0 & 0 & 0 & $\mathbf{0}$ & 0 & 0 & 0 & $\mathbf{0}$ \\
\hline Tricharaea & 0 & 0 & 1 & 0 & 0 & 0 & 1 & 0 & 0 & 0 & 0 & $\mathbf{0}$ & 0 & 0 & 0 & $\mathbf{0}$ \\
\hline Totales & & & & & & & 8 & & & & & $\mathbf{0}$ & & & & 2 \\
\hline
\end{tabular}


A las 48 horas de exposición, las principales especies que arribaron a los tejidos a la primera hora fueron Pekia (pantonella) intermutans con siete especímenes, Sarcofahrtiopsis sp, y Sarcodexia sp. con tres y Peckia sp con dos (Cuadro 4).

Cuadro 4. Total de especies de moscas capturadas a las 48 horas en el pns

\begin{tabular}{|c|c|c|c|c|c|c|c|c|c|c|c|c|c|c|c|c|}
\hline \multirow[t]{2}{*}{ ESPECIES DE MOSCAS } & \multicolumn{6}{|c|}{$\begin{array}{l}\text { PRIMERA HORA } \\
\text { (MIN) }\end{array}$} & & \multicolumn{4}{|c|}{$\begin{array}{l}\text { SEGUNDA } \\
\text { HORA } \\
(\text { MIN) } \\
\end{array}$} & \multicolumn{5}{|c|}{$\begin{array}{l}\text { TERCERA } \\
\text { HORA }\end{array}$} \\
\hline & 10 & 20 & 30 & 40 & 50 & & & 15 & 30 & 45 & 60 & & 20 & 40 & 60 & TOTAI \\
\hline SARCOPHAGIDAE & & & & & & & & & & & & & & & & \\
\hline Oxysarcodexia timida & 0 & 0 & 0 & 0 & 0 & 0 & 0 & 0 & 0 & 0 & 0 & $\mathbf{0}$ & 2 & 0 & 1 & 3 \\
\hline Pekia (pantonella) intermutans & 4 & 1 & 0 & 0 & 0 & 2 & 7 & 0 & 0 & 2 & 2 & 4 & 1 & 1 & 1 & 3 \\
\hline Pekia sp1 & 1 & 0 & 0 & 1 & 0 & 0 & 2 & 0 & 0 & 0 & 0 & $\mathbf{0}$ & 0 & 0 & 0 & $\mathbf{0}$ \\
\hline Sarcodexia sp & 0 & 0 & 0 & 0 & 0 & 1 & 1 & 0 & 2 & 1 & 1 & 4 & 0 & 0 & 0 & $\mathbf{0}$ \\
\hline Sarcofahrtiopsis sp & 0 & 0 & 0 & 0 & 2 & 1 & 3 & 0 & 1 & 0 & 0 & 1 & 0 & 0 & 0 & $\mathbf{0}$ \\
\hline Tricharaea & 0 & 0 & 0 & 0 & 0 & 0 & 1 & 0 & 0 & 0 & 1 & 1 & 0 & 0 & 0 & $\mathbf{0}$ \\
\hline & & & & & & & 14 & & & & & 12 & & & & 6 \\
\hline
\end{tabular}

A las 72 horas de exposición la especies que mayormente arribaron a los tejidos a la primera hora fueron Pekia (pantonella) intermutans con siete especímenes, en menor cantidad Pekia sp. y Villegasia postuncinata con un ejemplar cada una (Cuadro 5).

Cuadro 5. Total de especies de moscas capuradas a las 72 horas en el pns

\begin{tabular}{|c|c|c|c|c|c|c|c|c|c|c|c|c|c|c|c|c|}
\hline \multirow[t]{2}{*}{ ESPECIES+B2:R10 DE MOSCAS } & \multicolumn{6}{|c|}{$\begin{array}{c}\text { PRIMERA HORA } \\
\text { (MIN) }\end{array}$} & \multirow[t]{2}{*}{ TOTAL } & \multicolumn{4}{|c|}{$\begin{array}{c}\text { SEGUNDA } \\
\text { HORA (MIN) }\end{array}$} & \multirow{2}{*}{ TOTAL } & \multicolumn{3}{|c|}{$\begin{array}{c}\text { TERCER } \\
\text { A HORA } \\
(\text { MIN) }\end{array}$} & \multirow{2}{*}{ TOTAL } \\
\hline & 10 & 20 & 30 & 40 & 50 & 60 & & 15 & 30 & 45 & 60 & & 20 & 40 & 60 & \\
\hline \multicolumn{17}{|l|}{ SARCOPHAGIDAE } \\
\hline Pekia (pantonella) intermutans & 3 & 1 & 1 & 2 & 0 & 0 & 7 & 0 & 0 & 2 & 1 & 3 & 0 & 0 & 2 & 2 \\
\hline Pekia $\mathrm{sp} 1$ & 0 & 0 & 0 & 0 & 0 & 1 & 1 & 0 & 0 & 0 & 0 & $\mathbf{0}$ & 0 & 0 & 0 & $\mathbf{0}$ \\
\hline Sarcodexia $\mathrm{sp}$ & 0 & 0 & 0 & 0 & 0 & 0 & $\mathbf{0}$ & 1 & 1 & 0 & 0 & 2 & 0 & 0 & 0 & $\mathbf{0}$ \\
\hline Sarcofahrtiopsis sp & 0 & 0 & 0 & 0 & 0 & 0 & $\mathbf{0}$ & 0 & 0 & 0 & 0 & $\mathbf{0}$ & 0 & 0 & 1 & 1 \\
\hline Villegasia postuncinata & 0 & 0 & 0 & 0 & 1 & 0 & 1 & 0 & 0 & 0 & 0 & $\mathbf{0}$ & 0 & 0 & 0 & $\mathbf{0}$ \\
\hline Totales & & & & & & & 9 & & & & & 5 & & & & 3 \\
\hline
\end{tabular}


En los tejidos con 96 horas de exposición, las principales especies que arribaron a los tejidos en la primera hora fueron Sarcodexia sp con 12 especímenes, Pekia (pantonella) intermutans y Pekia sp con siete especímenes cada una, Boettcheria sp con seis, Helicobia sp. con cinco y Sarcofahrtiopsis sp2 cuatro especímenes (Cuadro 6).

Cuadro 6. Total de especies de moscas capuradas a las 96 horas en el pns.

\begin{tabular}{|c|c|c|c|c|c|c|c|c|c|c|c|c|c|c|c|c|}
\hline \multirow{2}{*}{$\begin{array}{l}\text { ESPECIES DE MOSCAS } \\
\text { SARCOPHAGIDAE }\end{array}$} & \multicolumn{6}{|c|}{$\begin{array}{l}\text { PRIMERA HORA } \\
\text { (MIN }\end{array}$} & \multirow{2}{*}{ TOTAL } & \multicolumn{4}{|c|}{$\begin{array}{c}\text { SEGUNDA } \\
\text { HORA (MIN) }\end{array}$} & \multirow[t]{2}{*}{ TOTAL } & \multicolumn{3}{|c|}{$\begin{array}{c}\text { TERCERA } \\
\text { HORA (MIN) }\end{array}$} & \multirow[t]{2}{*}{ TOTAL } \\
\hline & 10 & 20 & 30 & 40 & 50 & 60 & & 15 & 30 & 45 & 60 & & 20 & 40 & 60 & \\
\hline Blaesoxipha $\mathrm{Sp}$ & 0 & 0 & 1 & 0 & 0 & 0 & 1 & 0 & 0 & 0 & 0 & 0 & 0 & 2 & 0 & 2 \\
\hline Boettcheria Sp. & 1 & 2 & 3 & 0 & 0 & 0 & 6 & 1 & 1 & 2 & 3 & 7 & 4 & 4 & 0 & 8 \\
\hline Helicobia Sp. & 0 & 1 & 3 & 0 & 1 & 0 & 5 & 1 & 2 & 1 & 3 & 7 & 1 & 2 & 0 & 3 \\
\hline Oxysarcodexia timida & 0 & 0 & 0 & 0 & 0 & 0 & 0 & 0 & 1 & 0 & 0 & 1 & 0 & 0 & 0 & 0 \\
\hline Pekia (pantonella) intermutans & 0 & 0 & 5 & & 2 & 0 & 7 & 1 & 2 & 0 & 0 & 3 & 0 & 2 & 1 & 3 \\
\hline Pekia sp1 & 0 & 0 & 3 & 0 & 1 & 3 & 7 & 0 & 0 & 1 & 2 & 3 & 1 & 0 & 0 & 1 \\
\hline Sarcodexia sp & 1 & 6 & 2 & 1 & 2 & 0 & 12 & 1 & 3 & 2 & 7 & 13 & 3 & 4 & 0 & 7 \\
\hline Sarcofahrtiopsis sp1 & 0 & 0 & 1 & 0 & 1 & 1 & 3 & 0 & 1 & 0 & 1 & 2 & 0 & 0 & 0 & 0 \\
\hline Sarcofahrtiopsis sp2 & 1 & 0 & 1 & 0 & 1 & 1 & 4 & 0 & 0 & 1 & 1 & 2 & 0 & 1 & 0 & 1 \\
\hline Tricharaea $\mathrm{sp}$ & 0 & 0 & 0 & 0 & 0 & 1 & 1 & 0 & 0 & 0 & 0 & 0 & 0 & 0 & 0 & 0 \\
\hline Villegasia postuncinata & 0 & 1 & 0 & 0 & 0 & 0 & 1 & 0 & 0 & & 0 & 0 & 0 & 0 & 0 & \\
\hline Totales & & & & & & & 47 & & & & 38 & & & & & 25 \\
\hline
\end{tabular}

\section{DISCUSIÓN}

Las especies y los géneros más frecuentemente capturados en ambas áreas fueron: Pekia (Pantonella) intermutans (Thomson 1869), Sarcodexia sp., Boettcheria sp. Pekia sp. Helicobia sp. y Sarcofahrtiopsis sp2. Estos mismos géneros han sido recolectados en otros estudios similares (Buenaventura et al., 2009; Barbosa et al., 2010; Yepes-Gaurisas et al., 2013). Sin embargo, debido a la distribución de los mismos en los diferentes ecosistemas, las cantidades y la biodiversidad pudieran cambiar de un lugar a otro (Carvalho y Linhares, 2001; Mello-Patiu, 2016; De Souza y Von Zuben, 2016). Otra razón para explicar nuestro resultado puede ser el grado de humedad del cebo, las vísceras empleadas, la edad del cebo y la etapa de descomposición del tejido. También son factores importantes capaces de afectar el número, 
la composición de la edad de las poblaciones y el sexo de las moscas (Vogt y Woodburn, 1994). Por ejemplo, un estudio realizado en Argentina, por Aballay et al., (2011) en carcasas de cerdos, recolectaron 597 individuos, repartidos en cinco especies. Otro estudio realizado por Rodríguez y Salazar, (2014) en vísceras de res, colectaron un total de 127 ejemplares, cuatro de los cuales correspondieron a Sarcophagidae. De Souza y Von Zuben, 2016), en Brazil colectaron un total de 440 especímenes de la familia Sarcophagidae. En tanto Carvalho y Linhares, (2001) en São Paulo y, Mello-Patiu, (2016) en Colombia reportaron 21 y 102 especies de Sarcophagidae respectivamente.

La diferencia entre estos resultados, con los nuestros se puede deber a diversos factores como al tipo de cebo empleado, la vegetación del lugar, la metodología de captura empleada, la duración y la estacionalidad en la que se realizaron estos estudios (Andersonand y VanLaerhoven, 1996; Watson y Carlton, 2003; Barbosa et al., 2010; Kyerematen et al., 2012). No obstante, coincidimos con otros autores, que reportan que en el área boscosa se capturaron más moscas que en el área intervenida (Sousa et al., 2011; Vasconcelos et al., 2013; Carvalho et al., 2004).

Aunque la diferencia en nuestras capturas a nivel de especies no fue muy marcada, probablemente por la cercanía entre los sitios, debido a la influencia del área boscosa sobre el área intervenida, debido a que ambos sitios estuvieron separados por aproximadamente $50 \mathrm{~m}$. La mayor captura de moscas se realizó en el área boscosa, esto se puede deber a que este pudiera ser el medio natural donde se crían y reproducen estas moscas. En donde el ambiente presenta condiciones más favorables de temperatura y humedad y donde diversidad de sustratos en descomposición es mayor por la diversidad de mamíferos en el área.

En nuestro estudio, las especies que presentaron las mayores capturas en ambos tejidos fueron: Peckia (Pantonella) intermutans con $22.8 \%$ y $35.8 \%$ de las capturas, seguido de Sarcodexia sp. con $25.0 \%$ y $27.2 \%$, Helicobia sp con $10.8 \%$ y $6.4 \%$ Boettcheria sp con $9.7 \%$ y $16.2 \%$, Pekia sp $9.7 \%$ y $8.9 \%$. Linhares (1981) reportó que las moscas del género Helicobia sp fueron atraídas por la víscera de pollo, cadáveres de ratones 
$\mathrm{y}$ heces humanas. También presentaron un total rechazo a los asentamientos humanos.

Un estudio realizado por Sousa et al., (2011) en Brasil, con pulmón de res, reportaron la captura de 106 ejemplares de Pekia (Pantonella) intermutans la que resulto ser la especie es la más abundante en los bosques. Carvalho et al., (2004) también reportó esta especie como exclusiva de áreas boscosas. También ha sido reportada por Salviano, (1996) y por Souza y Linhares, (1997) en cerdos domésticos y en cadáveres humanos.

Por otro lado, las moscas del género Sarcodexia sp. son consideradas especies oportunistas que resultan atraídas por una variedad de sustratos, como cadáveres de cerdos y humanos (Oliveira et al., 2001; Barros et al., 2008). De acuerdo con Barros et al., (2008) la especie Sarcodexia lambens mostró una gran atracción por las vísceras de pollo y pescado, según otros estudios esta especie ha sido encontrada en gran proporción en cadáveres humanos.

La variación y abundancia de las especies de moscas, en los tejidos, parecen estar asociadas al área de estudio, las etapas de la descomposición de los tejidos y a la flora bacteria imperante en los mismos. En la (Figura 1) se destaca las dos principales especies Peckia (Pantonella) intermutans y Sarcodexia sp. que arribaron con mayor frecuencia a los tejidos durante el estudio.

En cuanto a la preferencia de las áreas, las moscas unas mostraron moderada preferencia por el área boscosa que por el área no boscosa, en algunas especies, debido a la cantidad en que fueron registradas. Aunque las áreas compartieron seis especies en común, es importante resaltar que cuatro especies (Sarcofahtiopsis sp1, Oxysarcodexia timida, Blaexosipha sp. y Villegasia puncinata) solo se registraron en el área boscosa. Resultados similares obtuvieron (Linhares, 1981: Días et al., 1984) en cuanto a la poca diversidad, al evaluar la preferencia de las moscas por las áreas urbanas, rurales y las boscosas, encontraron que las áreas urbanas y rurales no contienen una amplia variedad de sustratos que le puedan servir como alimento y/u oviposición a estas moscas, a 
diferencia del área boscosa. De acuerdo con Lopes, (1946) el género Oxisarcodexia sp es característico de la Región Neotropical y es el más grande en cuanto a número de especies, se desarrolla preferiblemente en heces. Este género es considerado de importancia en los estudios de entomología forense en Brasil debido a su abundancia, diversidad y hábitos alimenticios (Días et al., 1984, Carvalho y Linhares 2001; Barbosa et al., 2009).

En términos de sus preferencias de sus hábitos alimenticios, las moscas de la carne mostraron mayor preferencia por el corazón que por el hígado. En ambos tejidos se encontró muy poca diferencia en cuanto a la cantidad de moscas que fueron atraídas, no obstante en cuanto a su diversidad en el corazón se registraron las especies Blaexosipha sp. y Villegasia puncinata, que no fueron registradas en el hígado. Sin embargo, es importante mencionar que ambos tejidos permitieron registrar una moderada diferencia en cuanto la atracción en la diversidad $\mathrm{y}$ en el número de moscas, lo que a primera vista sugiere que ambos tejidos presentaron muy pocas diferencias en cuanto a su descomposición inicial y a lo largo del estudio. El corazón presentó muy pocas variaciones, en su inicio, en relación a su consistencia y en su descomposición característica, presentó una consistencia más firme que el hígado, debido a su musculatura fibrosa, mientras que el hígado fue más blando y esponjoso. Los fluidos de hígado empezaron a drenar, a partir del segundo día, lo que lo hizo que sus olores se disiparan mejor que los del corazón.

A continuación, cuantificamos el arribo de las principales especies de Sarcophagidae que llegaron a los tejidos. En el corazón se registró el mayor número de individuos. Se capturaron un total de 110 especímenes, mientras que en el hígado se capturaron 59 especímenes. Las especies más frecuentemente capturadas en el corazón fueron Pekia (Pantonella) intermutans y, Sarcodexia sp. y Boettcheria sp. En tanto que en el hígado se capturaron las mismas especies, aunque en menor cantidad (Cuadro 3). Inicialmente el hígado atrajo más moscas que el corazón.

A las 24 horas de exposición de los tejidos, en el caso del corazón, el 
mismo presentaba una consistencia más firme y su color era rojo pálido. La primera especie que arribó al mismo fue Peckia (Pantonella) intermutans, posteriormente continuaron arribando otros especímenes, aunque en muy bajos números. Contrario a lo reportado por Rodríguez y Salazar, (2014) quienes en su estudio con vísceras reporto a esta familia al segundo día de descomposición, cuando en nuestro estudio aparecieron ejemplares en el primer día de observación.

A las 24 horas de exposición de los tejidos, en uno de los intervalos de los 20 minutos, a la tercera hora de muestreo, en el corazón se observó la puesta de larvas de la especie de Pekia (Pantonella) intermutans a escasos segundos de haber llegado al tejido. Las pequeñas larvas eclosionaron y rápidamente se enterraron en el tejido. Por esta razón, es que esta especie pudiera ser considerada buena indicadora forense. Porque marcaría el tiempo aproximado de la muerte.

El hígado, presentaba un color rojo intenso, no obstante su color no había variado mucho con relación al día anterior. No se evidenciaba en el aire el olor a descomposición.

El tejido de corazón expuesto a las 48 horas, presentaba un color más pálido, rosado-cremoso, con una consistencia más blanda, se observó la presencia de burbujas producto de la liberación de los gases de la descomposición. En tanto que el hígado presentaba un color rojo oscuro y el olor empezaba a sentirse en el área. Las especies más capturadas en el mismo fueron: Pekia (Pantonella) intermutans con seis especímenes, seguido de Sarcofahrtiopsis sp1 con tres y Oxysarcodexia timida con dos. Algunos autores reportan que las Sarcophagidae presentan distintas preferencias alimentarias que van desde materia orgánica en descomposición, coprófagas, saprófagas, depredadores y parasitoides de abejas y avispas, parasitoides obligatorios y facultativos, pocas larvas causan miasis (Stevens et al., 2006; Moretti et al., 2009; Mello-Patiu y Luna-Días, 2010).

A las 72 horas de exposición, el corazón presentaba una consistencia pastosa, el color era cremoso pálido con zonas verdes, su olor era más intenso. Mientras que el hígado presentaba un color rojo oscuro, además 
presentaba burbujeo debido a la liberación de gases de la fermentación y con abundante líquido que drenaba al fondo de recipiente, con un fuerte olor a descomposición que se esparcía por toda el área, debido a la liberación de gases como sulfuro, amoniaco y nitrógeno, que son las sustancias químicas que atraen a las moscas. En este estado de la descomposición ambos tejidos atrajeron la mayor cantidad de moscas sarcófagas Pekia (pantonella) intermutans, seguido de Peckia y de Sarcodexia sp)

A las 96 horas de exposición, los tejidos se habían desintegrado casi en su totalidad, solo quedan restos amorfos y acuosos en el fondo de los recipientes. A pesar de presentar una gran emanación de líquido acuoso debido a la degradación de los mismos, los mismos no producían fuertes olores, por lo que, las moscas fueron disminuyendo cantidad y diversidad, con excepción de ciertas especies que persistieron hasta que finalizo el estudio. En el caso de corazón, el mismo presentaba un color crema-blanquecino pálido, el olor característico de la descomposición se había reducido drásticamente que casi no se percibía. Las especies que persistieron fueron: Sarcodexia sp, Boettcheria sp, Helicobia sp. y Peckia (pantonella) intermutans. Al cuarto día de descomposición de los tejidos, pese a que los olores de los tejidos no eran tan intensos como al inicio, las Sarcophagidae continuaron llegando a los tejidos, el mayor cantidad y diversidad de moscas. A esta altura los tejidos presentaron mayor cantidad moscas y diversidad de moscas. Contrario a lo reportado por Barbosa et al., (2010) quienes reportaron la mayor presencia en las etapas de hinchazón y descomposición.

Por lo menos, cuatro géneros de Sarcophagidae recogidos en este estudio pueden considerarse de importancia forense potencial debido a su arribo a los tejidos.

\section{CONCLUSIONES}

Cuatro géneros de Sarcophagidae capturadas en este estudio pueden ser consideradas que tienen potencial forense importante, debido a su constante arribo a los tejidos. 
Las especies que fueron frecuentemente capturadas en ambas áreas fueron Pekia (pantonella) intermutans, Sarcodexia sp, Boettcheria sp, Pekia sp, Helicobia sp y Sarcofahrtiopsis sp2.

Las especies que presentaron las mayores capturas en ambos tejidos fueron Peckia (pantonella) intermutans, seguida de Sarcodexia sp., Boettcheria $s p$, Pekia sp y Helicobia sp.

\section{REFERENCIAS}

Aballay, F., F. Fernández-Campón, P. Mulieri y S. Urquiza. 2011. Sarcophagidae (Diptera) de importancia forense en la puna de Catamarca, Argentina: ovoviviparidad como ventaja en condiciones de extrema aridez. Revista Sociedad Entomológica Argentina. 70: $255-266$.

Anderson, G. y S. VanLaerhoven 1996. Initial studies oninsect succession on carrion in southwestern. British Columbia. Journal of Forensic Sciences. 41: 617-625.

Anderson, G.S. 1995. The use of insects in death investigations: an analysis of cases in British Columbia over a five year period. Can. Soc. Journal of Forensic Science 28: 277-292.

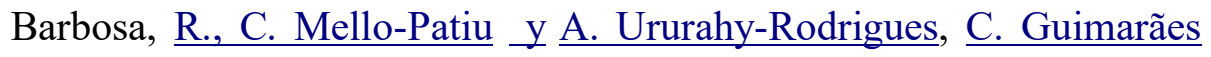
Barbosa 2010. Temporal distribution of ten calyptrate dipteran species of medicolegal importance in Rio de Janeiro, Brazil. Memórias do Instituto Oswaldo Cruz 105:191-8.

Barros, R.M., C.A. Mello-Patiu y J.R. Pujol-Luz. 2008. Sarcophagidae (Insecta, Diptera) associados à decomposição de carcaças de Sus scrofa Linnaeus (Suidae) em área de Cerrado do Distrito Federal, Brasil. Revista Brasilera de Entomología. 52: 606-609.

Buenaventura, E. y T. Pape. 2013. Revision of the new world genus Peckia Robineau-Desvoidy (Diptera: Sarcophagidae). Zootaxa 3622: 187. 
Buenaventura, E.R., G.C. Camacho, A.G. García y M. Wolff. 2009. Sarcophagidae (Diptera) de importancia forense en Colombia: claves taxonómicas, notas sobre su biología y distribución. Revista Colombiana de Entomología 35: 189-196.

Carvalho, L.M., P.J Thyssen, M.L Goff y A.X Linhares, 2004. Observations on the Succession Patterns of Necrophagous Insects on a Pig Carcass in an Urban Area of Southeastern Brazil. Journal of Forensic Medicine and Toxicology 5: 33-39.

Carvalho, L.M. y A.X. Linhares. 2001. Seasonality of insect succession and pig carcass decomposition in a natural forest area in southeastern Brazil. J. $\quad$ Forensic. $\quad$ Sci. 46, 604-608.

Dias, E.S., D. Neves y H.S. Lopes. 1984. Estudos sobre a fauna de Sarcophagidae (Diptera) de Belo Horizonte, Minas Gerais. I. Levantamento taxonômico e sinantrópico. Mem Inst Oswaldo Cruz 79:83-91.

Goff, M.L. 1991.Comparison of insect species associated with decomposing remains recovered inside dwellings and outdoors on the Island of Oahu, Hawaii. Journal of Forensic Science. 36: 748-753.

Kyerematen, R.A., B.A. Boateng y E. Twumasi. 2012. Insect diversity and succession pattern on different carrion types. Journal of Research in Biology. 2: 683-690.

Linhares, A.X. 1981. Synanthropy of Calliphoridae and Sarcophagidae (Diptera) in the city of Campinas, São Paulo, Brazil. Revista Brasileña de Entomología 25:189-215.

Lopes, H.S. 1946. Contribuição ao conhecimento das espécies do gênero Oxysarcodexia Townsend, 1917 (Diptera, Sarcophagidae). Boletim da Escola Nacional de Veterinária 1: 62-134.

Mello-Patiu, C.A. 2016. Family Sarcophagidae. Zootaxa. 1: 884-903 Mello-Patiu, C.A. y C. Luna-Dias. 2010. Myiasis in the Neotropical 
amphibian Hypsiboas beckeri (Anura: Hylidae) by a new species of Lepidodexia (Diptera: Sarcophagidae). Journal of Parasitology. 96: 685688.

Ministerio de Desarrollo Agropecuario (24 de junio de 1985). «Decreto No. 13 de 27 de mayo de 1980, por el cual se crea el Parque Nacional "Soberanía" en el área del Canal de Panamá»». Gaceta Oficial LXXXII (20.333).

Moretti, T.C., S.M. Allegretti, C.A. Mello-Patiu, A.M. Tognolo, O.B. Ribeiro y D.R. Solis, 2009. Occurrence of Microcerella halli (Engel) (Diptera, Sarcophagidae) in snake carrion in southeastern Brazil. Revista Brasileira de Entomologia 53:318-320.

Oliva, A. 1997. Insectos de interés forense de Buenos Aires (Argentina). Primera lista ilustrada y datos bionómicos. Rev. Mus. Argentino Ciencias Naturales, VII (2), 59 pp.

Oliveira, C.J., C.A. Mello y S.M. López. 2001. Dípteros muscóides associados a cadáveres humanos no local da morte no estado do Rio de Janeiro. Brasil. Bol. Mus. Nac., N.S., Zool. 464: 1-6.

Pape, T., V. Blagoderov y M.B. Mostovski. 2011. Order Diptera Linnaeus, 1758. In: Zhang Z-Q (Ed.) Animal biodiversity: an outline of higherlevel classification and survey of taxonomic richness. Magnolia Press, Auckland, 222-229.

Pape, T. y G.A. Dahlem. 2010. Sarcophagidae (Flesh flies). In: B. V Brown, A. Borkent, J. M. Cumming, D.M. Wood, N. E. Woodley \& M. A. Zumbado [Eds], Manual of Central American Diptera Vol. 2. NRC Research Press, Ottawa, Canada. pp. 1297-1335.

Pape, T. 1996. Catalogue of the Sarcophagidae of the World (Insecta: Diptera). Memoirs on Entomology, International 8: 1-558. Rodríguez, J.N. y J.L. Salazar. 2014. Sucesión de la entomofauna cadavérica a partir de un biomodelo con vísceras de res. Revista de la Facultad de Ciencias de la Salud. Universidad de Carabobo. 18: 35-39. 
Salviano, R.J., R.P. Mello, R.F. Santos, L.C. Beck y A. Ferreira. 1996. Calliphoridae (Diptera) associated with human corpses in Rio de Janeiro, Brazil. Entomol Vectores 3:145-146.

Sharannowski, B.J., E.G. Walker y S.G. Anderson. 2008. Insect succession and descomposition patterns on shaded and sunlit carrion Saskatchewan in three different seasons. Forensic Science International. 179: 219.

Shewell, G.E. 1987. Sarcophagidae. In: McAlpine, J.F., Peterson, B.V., Shewell, G.E., Teskey, H.J., Vockeroth, J.R. y Wood, D.M. (Eds.), Manual of Nearctic Diptera, Vol. 2. Research Branch Monograph 28. Agriculture Canada, Ottawa, pp. 1159-1186.

Sousa, J.R., M.C. Esposito y F.S. Carvalho-Filho. 2011. Diversity of Calliphoridae and Sarcophagidae (Diptera, Oestroidea) in continuous forest and gaps at different stages of regeneration in the Urucu oilfield in western Brazilian Amazonia. Revista Brasileira de Entomologia .54: 578-582.

Souza, A.M. y A.X. Linhares. 1997. Diptera and Coleoptera of potential forensic importance in southeastern Brazil: relative abundance and seasonality. Med. Vet. Entomol. 11:8-12.

Souza, de C.R. y C.J. Von Zuben. 2016. Synanthropy of Sarcophagidae (Diptera) in southeastern Brazil. Neotrop Entomol. 45:637-641

Stevens, J.R., J.F. Wallman, D. Otranto, R. Wall y T. Pape. 2006. The evolution of myiasis in humans and other animals in the Old and New Worlds (part II): biological and life-history studies. Trends in Parasitology, 22: 181-188.

Vasconcelos, S.D., T.M. Cruz, R.L. Salgado y P.J. Thyssen. 2013. Dipterans associated with a decomposing animal carcass in a rainforest fragment in Brazil: notes on the early arrival and colonization by necrophagous species. Journal of Insect Science 13: 111 . 
Vogt, W.G. y T.L. Woodburn 1994. Effects of bait age on the number, sex, and age composition of Lucilia cuprina (Wiedmann) (Diptera: Callihoridae) in Western Autralian blowfly traps. Australian Journal of Experimental Agriculture. 34: 595-600. Watson, E. y C. Carlton 2003. Spring succession of necrophilous insects on wildlife carcasses in Louisiana. Journal of Medical Entomoloy. 40: 338-347.

Wells, J., T. Pape y F.A Sperling. 2001. DNA-based identification and molecular systematics of forensically important Sarcophagidae (Diptera). Journal Forensic Science 46: 1098-1102.

Yepes-Gaurisas, D., J.D. Sánchez-Rodríguez, C. A. de Mello-Patiu, M. Wolff. 2013. Synanthropy of Sarcophagidae (Diptera) in La Pintada, Antioquia-Colombia. Biología Tropical. 61:1275-1287.

Recibido 21 de febrero de 2020, aceptado 23 de marzo de 2020. 